\title{
Percutaneous pulmonary valve implantation in small conduits: A multicenter experience
}

\author{
Sebastien Hascoet a,b,c, José Diogo Martins ${ }^{\mathrm{d}}$, Haysam Baho ${ }^{\mathrm{e}}$, Saule Kadirova ${ }^{\mathrm{f}}$, Fatima Pinto ${ }^{\mathrm{d}}$, Florent Paoli ${ }^{\mathrm{g}}$, \\ Fadi Bitar ${ }^{\mathrm{h}}$, Abdelfatah abu Haweleh ${ }^{\mathrm{i}}$, Anselm Uebing ${ }^{\mathrm{j}}$, Philippe Acar ${ }^{\mathrm{a}}$, Olivier Ghez ${ }^{\mathrm{j}}$, Alain Fraisse ${ }^{\mathrm{j}, *}$ \\ a Hôpital des enfants, Cardiologie pédiatrique, Centre de Compétence Malformations Congénitales Complexes M3C, CHU Toulouse, 31100 Toulouse, France

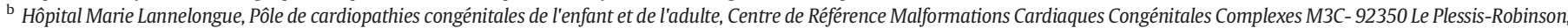 \\ Faculté de Médecine Paris-Sud, Université Paris Sud, Université Paris-Saclay, France \\ ' Inserm/UPS UMR 1048 - I2MC, CHU Toulouse, Toulouse, France \\ d Pediatric Cardiology Department, Hospital de Santa Marta, CHLC, Lisboa, Portugal \\ e King Faycal Specialist Hospital, Jeddah, Saudi Arabia \\ ${ }^{\mathrm{f}}$ National Research Cardiac Surgery Center, Astana, Kazakhstan \\ g La Timone Hospital, Marseille, France \\ ${ }^{h}$ American University of Beirut Medical Center, Beirut, Lebanon \\ ${ }^{i}$ Queen Alia Heart Institute. Amman, Jordan \\ ${ }^{\mathrm{j}}$ Royal Brompton and Harefield Hospitals Trust, London, UK
}

\section{A R T I C L E I N F O}

\section{Article history:}

Received 11 January 2017

Received in revised form 25 September 2017

Accepted 1 December 2017

Available online 5 December 2017

Keywords:

Percutaneous pulmonary valve implantation

Congenital heart defects

Children

\begin{abstract}
A B S T R A C T
Background: Guidelines allow percutaneous pulmonary valve implantation (PPVI) in conduits above $16 \mathrm{~mm}$ diameter. Balloon dilatation of a conduit to a diameter $>110 \%$ of the original implant size is also not recommended. We analyzed patients undergoing PPVI in such conditions.

Methods and results: Nine patients (May 2008-July 2016) from 8 institutions underwent PPVI in conduits $<16 \mathrm{~mm}$ diameter. Five patients with $16-18 \mathrm{~mm}$ conduit diameter underwent PPVI after over-expansion of the conduit $>110 \%$. Mean age and weight of the 14 patients was 12.1 ( 7.7 to 16 ) years and 44.9 (19 to 83 ) $\mathrm{kg}$. Median conduit diameter at PPVI was 12 (10 to 17) mm. Median systolic right ventricular pressure was 70 (40 to 94) $\mathrm{mm} \mathrm{Hg}$. Procedure was successful in all cases. A confined conduit rupture occurred in 7 patients (50\%) and was treated with covered stent in 6 . One patient experienced dislocation of 2 pulmonary artery stents that were parked distally. The post-implantation median systolic right ventricular pressure was 36 (28 to 51) $\mathrm{mm} \mathrm{Hg}$. A fistula between right-ventricle outflow and aorta was found in one patient, secondary to undiagnosed conduit rupture. This was closed surgically. After a median follow-up of 20.16 (6.95 to 103.61) months, all the patients are asymptomatic with no significant RVOT stenosis.

Conclusions: PPVI is feasible in small conduits but rate of ruptures is high. Although such ruptures remain contained and can be managed with covered stents in our experience, careful selection of patients and high level of expertise are necessary. More studies are needed to better assess the risk of PPVI in this population.
\end{abstract}

(C) 2017 Elsevier B.V. All rights reserved.

\section{Introduction}

Percutaneous pulmonary valve implantation (PPVI) has emerged as an alternative to surgery for reconstruction of the right ventricle outflow tract (RVOT). Since the first PPVI in 2000 [1], multiple studies have confirmed its safety and efficacy [2-9]. The Melody valve (Medtronic Inc., Minneapolis, MN, USA) has been the first valve inserted percutaneously in humans and obtained European certification (CE) in

\footnotetext{
* Corresponding author at: Royal Brompton \& Harefield NHS Foundation Trust, Sydney Street, London SW3 6NP, UK.

E-mail address: A.Fraisse@rbht.nhs.uk (A. Fraisse).
}

2006 as well as approval for use in the USA in 2010. Mid-term outcome is good with regards to hemodynamic evolution, functional status and device durability $[2-4,7,10]$. Nevertheless guidelines recommend its use in conduits with nominal diameter equal to or above $16 \mathrm{~mm}$ $[7,11]$. Moreover, overdilation of the conduit diameter $>110 \%$ of the nominal diameter (original implant size) prior to PPVI is not recommended (www.medtronic.com/safety-info-html). PPVI in children with small weight has previously been performed $[8,12,13]$. But little is known about PPVI efficiency in small and/or over-expanded conduits. A-priori issues were raised with regards to conduit rupture risk [14] and residual gradient across the valve [15]. Thus we analyzed outcome of patients with off-label PPVI in small and over-expanded conduits. 


\subsection{Study design}

Between November 2008 and July 2016, 14 patients who underwent off-label PPVI in conduits below $16 \mathrm{~mm}$ and/or in conduits below or equal to $18 \mathrm{~mm}$ with a final Melody valve to tube diameter ratio above $110 \%$ were retrospectively analyzed. Procedures were performed in 8 centers (Royal Brompton hospital, England, $\mathrm{n}=4$; CHU La Timone, Marseille, France, $\mathrm{n}=3$; Lisbon, Portugal, $\mathrm{n}=2$; CHU Toulouse, France, $\mathrm{n}=1$; Astana, Kazakhstan, $\mathrm{n}=1$; Amman, Jordan, $\mathrm{n}=1$; Jeddah, Saudi Arabia, $\mathrm{n}=1$; Beirut, Lebanon, $\mathrm{n}=1$ ).

Patients were considered for PPVI if they had congenital heart disease requiring previous RVOT surgery, with an indication for pulmonary valve replacement according to current indications and practices [11]. Patients were discussed and approved for off-label PPVI after a joint cardiac surgical conference. Only patients with "expandable" conduits such as Contegra conduit or homograft or native outflow tract were considered suitable for off-label PPVI. Otherwise, patients with "non-expandable" conduits such as Hancock conduits below $16 \mathrm{~mm}$ were not suitable for off-label PPVI and surgical PV replacement was considered. PPVI was contra-indicated in case of active infection or coronary artery compression during balloon testing. Patients with failure attempt of PPVI were not included in this study. The study protocol conforms to the ethical guidelines of the 1975 declaration of Helsinki. Written informed consent for the procedure was obtained from al patients and/or parents before PPVI. The off-label indication of PPVI was explained to the family together with the alternative possibility of surgical valve replacement. The risk of conduit rupture and the potential for emergency surgery were also clearly stated.

\subsection{Procedure}

Melody valves were used for PPVI. The Melody valve is made of a bovine jugular valved vein (Contegra Pulmonary Valved conduit, Medtronic Inc., Minneapolis, MN, USA) sutured within a Cheatham-Platinium stent (CP stent, NuMED Inc., Hopkinton, $\mathrm{NY}$ ). The valve was manually crimped and implanted through a dedicated delivery system (Ensemble Transcatheter Delivery System, Medtronic Inc., Minneapolis, MN, USA). It consists of a balloon-in-balloon (BiB, NuMED Inc., Hopkinton, NY) catheter delivery system with a retractable sheath that covers the Melody valve once it is crimped over the balloon. The outer balloon is available in 3 sizes: 18, 20 and $22 \mathrm{~mm}$ in diameter.

In view of a potential unconfined conduit rupture requiring covered stent implantation in emergency, all the patients had a pre-procedure computerized tomodensitometry to assess proximity of the coronary arteries from the conduit. RVOT calcification was estimated on pre-procedure CT scan in each patient, using the following descriptive, semiquantitative approach: Grade $0=$ no calcification, $1=$ mild calcification, $2=$ moderate calcification that is not circumferential, $3=$ moderate calcification that is circumferentia or heavy calcification that is not circumferential, and $4=$ heavy calcification that is circumferential. All procedures were achieved under general anesthesia. Regarding the risk for conduit rupture during staged balloon dilation of the conduit, surgical back-up was organized on a systematic basis. PPVI was performed by a local specialist assisted by the primary investigator (A.F.) in 13 of the 14 cases. Percutaneous trans-femoral access was used in 13 patients. PPVI was done on a one-stage procedure in all cases. Direct invasive hemodynamic measurements and angiographic assessment were made in all patients before and after valve deployment. Minimal internal tube diameter was measured. Progressive balloon dilation of the conduit with high (Mullins, NuMED Inc., Hopkinton, New-York, USA) or ultra-high (Atlas or Atlas Gold, Bard, Tempe, AZ, USA) pressure balloons was performed, through a 14 French long Mullins sheath that was advanced immediately below the conduit. A balloon 2 to $4 \mathrm{~mm}$ larger than the conduit diameter was initially used. RVOT angiography was performed after every balloon dilation, to rule-out any conduit rupture. In the absence of rupture, step-by step balloon dilation of the conduit was performed using $2 \mathrm{~mm}$ diameter increment until the diameter of the intended pre-stent was reached. Aortic root angiogram was then performed during this latest balloon dilation to exclude coronary compression in lateral and either right-anterior or left-anterior and cranial projection. In case a confined conduit rupture was diagnosed during progressive balloon dilation of the conduit, a covered stent was immediately crimped on a BIB balloon (NuMED Inc., Hopkinton, New-York, USA) of the same diameter or $2 \mathrm{~mm}$ larger than the last balloon used for RVOT dilation. If coronary artery testing was already performed, the covered stent was implanted in the RVOT to treat the conduit rupture. If coronary testing was not yet performed, subsequent balloon dilation of the RVOT was performed with the same or a slightly ( $2 \mathrm{~mm}$ ) larger balloon than the last one used for RVOT dilation, with concomitant aortic root angiogram. Covered stent was immediately implanted after coronary compression testing. After pre-stenting of the landing zone, Melody valve was implanted through the Ensemble delivery system using the standardized method [11].

At the time of the procedure, no patient presented ongoing infection on either clinical or biological exams. All patients received antibiotics bolus $(25 \mathrm{mg} / \mathrm{kg}$ intravenous cefazolin) and anticoagulation (100 UI/ $\mathrm{kg}$ intravenous heparin) at the beginning of the procedure. Prophylaxis and heparin were repeated if the procedure was prolonged, to maintain the ACT $>250$. After PPVI, oral aspirin (100-250 mg once a day) was prescribed life-long. Antibiotic prophylaxis and non-specific prevention measures were recommended following guidelines [16]

\subsection{Follow-up}

Outcome was assessed in September 2017 in all patients. All local investigators were contacted to obtain clinical and echo data at last visit.

\subsection{Statistical analysis}

For each patient, we collected demographic characteristics, procedural hemodynamic data, technical details, results and follow-up. Statistical analyses were performed using Stata 11.2 software (Statacorp, Texas, USA). Data are summarized as mean (Standard Deviation), minimal and maximal values. The Shapiro-Wilks test did not reject normality of distribution of continuous variables. Categorical variables were summarized as number and percentages. Pre and post procedural hemodynamic data were compared using a Wilcoxon matched-pairs signed rank test. Fischer Exact and Mann-Whitney tests were used to compare variables of interest among patients with versus without conduit rupture after balloon predilation. Reported $\mathrm{P}$-values are two sided. Values of $\mathrm{P}<0.05$ were considered statistically significant.

\section{Results (Tables 1-3, Fig. 1)}

A total of 14 patients were included at a mean age of $12.1 \pm 3.0$ years old between November 2008 and July 2016.

Congenital heart defects were cono-truncal defects in 10 cases (71.4\%), congenital aortic valve disease with Ross surgery in 2 cases (14.3\%), transposition of the great arteries in 1 case (7.1\%) and pulmonary valve stenosis in 1 case (7.1\%). All patients had a right ventricle to pulmonary artery conduit. A homograft had been used in 10 cases (71.4\%), a Contegra (Contegra Pulmonary Valved conduit, Medtronic Inc., Minneapolis, MN, USA) in 3 cases (21.4\%) and a non valved tube in 1 case (7.1\%). Patient demographics data are presented in Table 1.

Procedural data are displayed in Table 2. PPVI was performed in a one-stage procedure in all cases. A trans-femoral approach was used in 13 cases whereas a left internal jugular vein approach was required in one patient. Predilation of the conduit was performed in all cases with simultaneous coronary artery angiogram. Pre-stenting of the conduit was performed in 13 cases (92.9\%). Only one patient with a $15 \mathrm{~mm}$ homograft had no prestenting at the beginning of the experience. Two stents were implanted in the RVOT conduit in 4 cases (28.6\%). Two

Table 1

Demographics and Diagnostic in patients undergoing percutaneous pulmonary valve implantation in small or overdilated conduits.

\begin{tabular}{|c|c|}
\hline Patients & $(\mathrm{n}=14)$ \\
\hline Age, years & 12.1 (3.0) 7.7-16 \\
\hline Weight, kg & $44.9(18.3) 19-83$ \\
\hline \multicolumn{2}{|l|}{ Congenital heart diseases } \\
\hline Commun arterial trunk & $5(35.7 \%)$ \\
\hline Tetralogy of fallot with pulmonary atresia & $3(21.4 \%)$ \\
\hline Tetralogy of fallot with pulmonary stenosis & $1(7.1 \%)$ \\
\hline Pulmonary valve agenesis & $1(7.1 \%)$ \\
\hline Ross procedure & $2(14.3 \%)$ \\
\hline Transposition of the great arteries & $1(7.1 \%)$ \\
\hline Pulmonary valve stenosis & $1(7.1 \%)$ \\
\hline \multicolumn{2}{|l|}{ Number of surgery } \\
\hline 1 & $10(71.4 \%)$ \\
\hline 2 & $4(28.6 \%)$ \\
\hline \multicolumn{2}{|l|}{ Right ventricle outflow tract } \\
\hline Homograft & $10(71.4 \%)$ \\
\hline Valved conduit (Contegra) & $3(21.4 \%)$ \\
\hline Non valved conduit & $1(7.1 \%)$ \\
\hline \multicolumn{2}{|l|}{ Conduit diameter at implantation, mm } \\
\hline 12 & $1(7.1 \%)$ \\
\hline 13 & $1(7.1 \%)$ \\
\hline 14 & $4(28.6 \%)$ \\
\hline 15 & $3(21.4 \%)$ \\
\hline 16 & $2(14.3 \%)$ \\
\hline 17 & $2(14.3 \%)$ \\
\hline 18 & $1(7.1 \%)$ \\
\hline \multicolumn{2}{|l|}{ Conduit diameter at catheterization, $\mathrm{mm}$} \\
\hline 10 & $2(14.3 \%)$ \\
\hline 11 & $4(28.6 \%)$ \\
\hline 12 & $2(14.3 \%)$ \\
\hline 13 & $2(14.3 \%)$ \\
\hline 14 & $3(21.4 \%)$ \\
\hline 16 & $1(7.1 \%)$ \\
\hline
\end{tabular}

Data are presented as frequency (\% of total patients in the column) or mean (Standard Deviation) minimum-maximum. 
Table 2

Procedural details for the 14 patients undergoing PPVI in small or overdilated conduits.

RV systolic pressure, $\mathrm{mmHg}$
RV to Aorta systolic pressure ratio, \%
Predilation balloon
Atlas Gold
Mullins
Cordis

Predilation diameter, $\mathrm{mm}$

\begin{tabular}{|c|c|c|}
\hline RV systolic pressure, mmHg & & 70 (16) 40-94 \\
\hline RV to Aorta systolic pressure ratio, \% & & 71 (18) 49-115 \\
\hline Predilation balloon & & \\
\hline Atlas Gold & & $9(64.3 \%)$ \\
\hline Mullins & & $4(28.6 \%)$ \\
\hline Cordis & & $1(7.1 \%)$ \\
\hline Predilation diameter, mm & 16 & $1(7.1 \%)$ \\
\hline & 18 & $1(7.1 \%)$ \\
\hline & 20 & $6(42.9 \%)$ \\
\hline & 22 & $6(42.9 \%)$ \\
\hline Largest balloon to nominal conduit diameter ratio, \% & & 137 (16) 107-167 \\
\hline Largest balloon - nominal conduit diameter, mm & & $5.4(1.9) 1.0-8.0$ \\
\hline Largest balloon to measured conduit diameter ratio, \% & & 169 (30) 119-200 \\
\hline Largest balloon - measured conduit diameter, mm & & 8.1 (2.7) $2.5-11.0$ \\
\hline Conduit rupture & & $7(50.0 \%)$ \\
\hline Prestenting before PPVI & & $13(92.9 \%)$ \\
\hline Number of stents implanted before PPVI & 0 & $1(7.1 \%)$ \\
\hline & 1 & $9(64.3 \%)$ \\
\hline & 2 & $4(28.6 \%)$ \\
\hline Type of stent & & \\
\hline CP 8-Zig stent & & $7(41.2 \%)$ \\
\hline Covered CP 8-Zig stent & & $6(35.3 \%)$ \\
\hline ev3 intrastent LDmax & & $3(17.6 \%)$ \\
\hline Andrastent & & $1(5.9 \%)$ \\
\hline Pulmonary valve diameter, mm & 18 & $2(14.3 \%)$ \\
\hline & 20 & $1(7.1 \%)$ \\
\hline & 22 & $11(78.6 \%)$ \\
\hline Valve - predilation balloon diameter, $\mathrm{mm}$ & 0 & $8(57.1 \%)$ \\
\hline & 2 & $6(42.9 \%)$ \\
\hline Valve to nominal tube diameter ratio, \% & & 143 (19) $120-183$ \\
\hline Valve to measured tube diameter ratio, \% & & 176 (31) 129-220 \\
\hline Severe procedural complications & & $0(0 \%)$ \\
\hline
\end{tabular}

Data are presented as frequency (\% of total patients in the column) or mean (Standard Deviation) minimum-maximum. RV, right ventricle; PPVI, percutaneous pulmonary valve implantation.

patients had associated lesions of the pulmonary artery bifurcation that were treated by balloon dilation and stent implantation during the PPVI procedure.

PPVI was successful in all cases. Mean systolic right ventricle pressure decreased from 70 to $36 \mathrm{~mm} \mathrm{Hg}(\mathrm{P}=0.002)$. Mean systolic right ventricle to systolic aortic pressure ratio decreased from $71 \%$ to $33 \%(\mathrm{P}=0.002)$. Final systolic right ventricle to systolic aortic pressure ratio was below $50 \%$ in all cases. Seven patients (50.0\%) experienced confined conduit rupture after predilation (Fig. 1). Covered stenting was performed and sufficient in 6 cases, with no residual leak on post implantation angiogram. In the last case, a tear at the lower extremity of the conduit was not diagnosed during the procedure because a fistula

Table 3

Comparison of patients with and without conduit rupture during PPVI.

\begin{tabular}{|c|c|c|c|}
\hline & $\begin{array}{l}\text { Conduit } \\
\text { rupture }\end{array}$ & $\begin{array}{l}\text { No conduit } \\
\text { rupture }\end{array}$ & P-value \\
\hline & $(\mathrm{n}=7)$ & $(\mathrm{n}=7)$ & \\
\hline Age, years & $12.2(3.6)$ & $12.0(2.6)$ & 0.9 \\
\hline Weight, kg & $52.5(21.4)$ & $37.3(11.4)$ & 0.2 \\
\hline Type of conduit & & & 0.2 \\
\hline Homograft & 6 & 4 & \\
\hline Contegra & 0 & 3 & \\
\hline Non valved conduit & 1 & 0 & \\
\hline Nominal conduit diameter, mm & $15.4(2.1)$ & $14.6(1.0)$ & 0.4 \\
\hline Conduit diameter at PPVI, mm & $12.6(2.3)$ & $12.1(1.5)$ & 0.6 \\
\hline Initial RV systolic pressure, mm Hg & $69(13)$ & $70(20)$ & 0.8 \\
\hline Initial RV to Aorta systolic pressure ratio,\% & $77(20)$ & $66(17)$ & 0.2 \\
\hline Balloon to nominal conduit diameter ratio, \% & $135(15)$ & $140(18)$ & 0.3 \\
\hline Balloon - nominal conduit diameter, mm & $5.1(1.5)$ & $5.7(2.4)$ & 0.3 \\
\hline Balloon to measured conduit diameter ratio, \% & $167(31)$ & $171(30)$ & 0.9 \\
\hline Balloon - measured conduit diameter, mm & $7.9(2.7)$ & $8.2(3.0)$ & 0.8 \\
\hline
\end{tabular}

Data are presented as frequency (\% of total patients in the column) or mean (Standard Deviation). RV, right ventricle; PPVI, percutaneous pulmonary valve implantation.

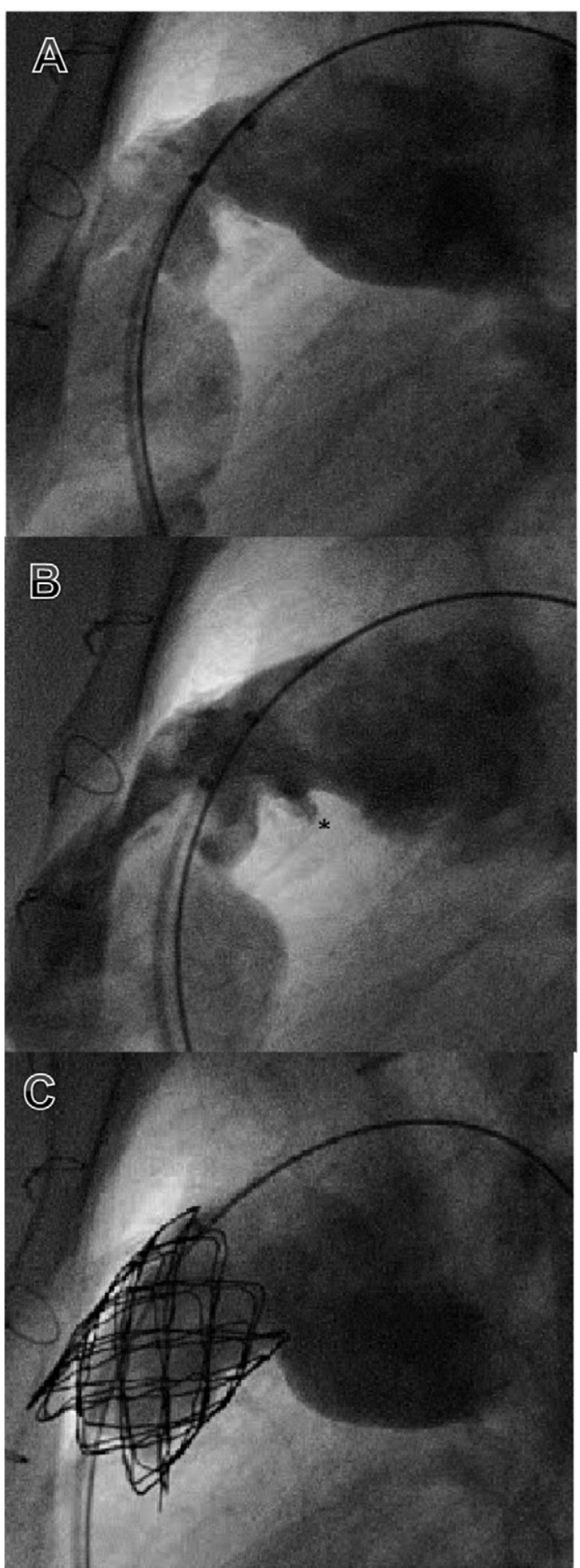

Fig. 1. Lateral angiogram showing a severely stenosed (10 mm diameter whereas initial nominal diameter was $16 \mathrm{~mm}$ ) homograft in the right ventricle outflow tract (A). After balloon dilation, confined rupture $\left(^{*}\right)$ is seen $(B)$. Control angiogram of the right ventricle outflow demonstrates an excellent result after a $22 \mathrm{~mm}$ Melody valve implantation (C).

developed between the RVOT and the aortic root. Then, no contrast extravasation was seen outside of the conduit during the post-dilatation angiogram in the RVOT. This fistula was clinically well-tolerated but because of significant shunt it was decided to close it. This fistula was surgically closed 14 months after PPVI as the proximity of the aortic end of the fistula to the right coronary ostium rendered it unsuitable for transcatheter closure. In another patient, bifurcation stenting was initially 
performed because of significant stenosis. Unfortunately, both stents embolized during PPVI. They were successfully "parked" in the distal pulmonary artery branches and no further intervention was done at the level of the bifurcation as right ventricular systolic pressure was $35 \mathrm{~mm} \mathrm{Hg}$ and there was only mild residual stenosis on angiography at the origin of both pulmonary artery branches. Comparison of variables among patients with versus without conduit rupture after balloon dilation is reported in Table 3. There was a trend towards more homografts in patients with conduit rupture. Calcification score was not related to conduit rupture. It was available in 12 patients including 6 with rupture and 6 without. Among the 4 cases with a calcification score at 4, 2 experienced conduit rupture. The score was 3 or 4 in only 4 of the patients who experienced conduits rupture whereas all 6 cases with no conduit rupture had a score of 3 or 4 .

Median follow-up duration was 20.16 (6.95 to 103.61) months. No patient was lost to follow-up. No pulmonary valve replacement, infective endocarditis or death was reported. No pulmonary valve regurgitation was observed in 10 patients (71.4\%) at last follow-up. A trivial to mild pulmonary valve regurgitation was reported in 4 patients (28.6\%). Mean peak pulmonary valve gradient at last follow-up was $23.8 \mathrm{~mm} \mathrm{Hg}$. All patients were in NYHA 1 functional status.

\section{Discussion}

In this multicenter cohort study, we observed that off-label PPVI could be satisfactorily performed in small conduits measuring $<16 \mathrm{~mm}$ of nominal diameter and in conduits between 16 and $18 \mathrm{~mm}$ diameter that could be over expanded above $110 \%$. Conduit rupture occurred in $50 \%$ of cases but was always confined in our experience. Such conduit ruptures with limited collection did not compromise the patients and were efficiently treated with covered stents, with the exception of a fistula between aortic root and RVOT that was treated surgically. Finally, before PPVI was performed, most of the patients (10 out of 14) had only one previous surgical procedure, during neonatal period or infancy.

Few data are available with regard to PPVI in small conduits. In a previous report including 25 patients weighting $<30 \mathrm{~kg}$ and undergoing PPVI, only 4 patients had a conduit below $16 \mathrm{~mm}$ diameter. No complication was reported but only $18 \mathrm{~mm}$ Melody valves were implanted [13]. The relevance of PPVI in small conduits was recently questioned by Holzer and Hijazi. They stated that only patients with a RVOT conduit larger than $16 \mathrm{~mm}$ are suitable for PPVI [15]. Moreover, in addition to the risk for conduit rupture, higher residual RVOT gradients following PPVI in smaller bioprosthesis were observed in another recent study [17]. In our patient population, we speculated that there would be significant residual RVOT gradient if the conduits were not optimally expanded. With a $22 \mathrm{~mm}$ Melody valve diameter in $76.9 \%$ of the patients, we aimed to implant the largest possible valve. To achieve this goal, "aggressive" conduit predilation was performed with a mean balloon to nominal conduit diameter ratio of $137 \%$ (up to $167 \%$ ) and a mean balloon to measured conduit diameter ratio of $169 \%$ (up to $200 \%$ ). This predilation step was also crucial to accurately assess the risk of coronary artery compression with a balloon diameter similar to the intended Melody valve diameter [18,19]. Non-compliant high and ultra-high pressure balloons were used and best suited for this purpose [15]. However in some cases conduit rupture occurred at an early stage of predilation. Optimal coronary testing using a much higher balloon diameter after conduit rupture may be life threatening by dramatically increasing the collection outside of the conduit. In view of this, preprocedure computerized tomodensitometry was performed on a systematic basis to potentially exclude any case with risk factor for coronary compression (single origin of the coronary arteries, coronary artery course close to the RVOT conduit...) $[18,19]$. Although no patient was excluded in our study, we would refer to surgery any case at risk for coronary artery compression on the pre-procedure computerized tomodensitometry. Following our strategy to favor large landing zones for PPVI, final hemodynamic and outcome were nicely improved. All patients had final systolic right ventricle to aorta pressure ratio below $50 \%$.

In the present study conduit rupture was observed in $50 \%$ of cases, much higher than the 4 to $9 \%$ incidence previously reported in consecutive PPVI [14,20,21]. They were mainly observed with homografts, as previously reported [14]. However, the risk of death and/or emergency surgery is not fully clarified after conduit rupture. Only one previous study reported 6 patients undergoing rescue surgeries after PPVI, including 2 cases after conduit rupture. Unfortunately, this study collected patients during early experience with PPVI (2000-2007) and covered stent implantation was apparently not attempted in those 2 cases. In a recent study by Bishnoi et al., 58 cases of conduits tears treated with covered stent were analyzed. No emergency surgery was needed and only 4 patients (7\%) had uncontained conduit rupture necessitating pleurocentesis and chest tube placement in 2 of them. Treatment with covered stent was successful in all cases but one. In this latest case the uncontained tear was stabilized by covered stent implantation but because of slow residual leak the patient was eventually operated 3 days after catheterization [20]. Similarly in our patient's population, we speculated that conduit rupture would be confined in the majority of cases because surgically implanted conduits are surrounded by fibrosis that may contain the collection outside of the conduit. In the present study, conduit ruptures and tears were indeed confined and successfully treated with covered stent implantation, with the exception of the patient who developed an aorta-to-RVOT fistula. However, we followed a rigorous protocol in all our cases. First, we managed to have a covered stent already crimped on a balloon and ready to be implanted at the time of conduit predilation. Second, surgical back-up was immediately available in case of any catastrophic conduit rupture, uncontained and uncontrolled even after covered stent implantation [14,20]. Third, conduit predilation was performed through a very progressive and gradual balloon dilation of the conduit. We always started predilation with a balloon of no $>125 \%$ of the narrowest diameter as this is recommended [15]. Further gradual expansion of the conduit was performed with $2 \mathrm{~mm}$ increment balloon diameter, until the intended predilation diameter was achieved to test the risk for coronary compression with a concomitant aortic root angiogram. Finally, RVOT angiogram was performed after every balloon dilation. With such protocol no patient was compromised by conduit rupture and no transfusion or chest tube drainage was required.

Our study has several limitations. The small number of patients precluded statistical analysis of risk factors for conduit rupture. There may also be selection bias and information recall bias which are standard limitations associated with any retrospective study. Finally, only Melody valves were used in this study. More recently, other devices such as the Sapien valve have become available for PPVI and could have also been used in smaller conduits with a similar approach [15].

\section{Conclusion}

Off-label PPVI is feasible in small conduits with good hemodynamic results. However, it is associated with an important risk of conduit rupture. Although we could successfully manage those contained ruptures with covered stents careful pre-procedure selection of patients and a high level of expertise are necessary to perform PPVI in this setting. More studies are needed before any definitive conclusion can be made regarding the risk of PPVI in this patient population.

\section{Funding}

None.

\section{Potential conflict of interest}

Alain Fraisse is a consultant and proctor for Medtronic Inc. Other authors have no conflict of interest. 


\section{References}

[1] P. Bonhoeffer, Y. Boudjemline, Z. Saliba, J. Merckx, Y. Aggoun, D. Bonnet, et al., Percutaneous replacement of pulmonary valve in a right-ventricle to pulmonary-artery prosthetic conduit with valve dysfunction, Lancet 356 (2000) 1403-1405.

[2] A. Fraisse, P. Aldebert, S. Malekzadeh-Milani, J.B. Thambo, J.F. Piechaud, P. Aucoururier, et al., Melody (R) transcatheter pulmonary valve implantation: results from a French registry, Arch. Cardiovasc. Dis. 107 (2014) 607-614.

[3] S. Khambadkone, L. Coats, A. Taylor, Y. Boudjemline, G. Derrick, V. Tsang, et al., Percutaneous pulmonary valve implantation in humans: results in 59 consecutive patients, Circulation 112 (2005) 1189-1197.

[4] P. Lurz, L. Coats, S. Khambadkone, J. Nordmeyer, Y. Boudjemline, S. Schievano, et al Percutaneous pulmonary valve implantation: impact of evolving technology and learning curve on clinical outcome, Circulation 117 (2008) 1964-1972.

[5] E.M. Zahn, W.E. Hellenbrand, J.E. Lock, D.B. McElhinney, Implantation of the melody transcatheter pulmonary valve in patients with a dysfunctional right ventricular outflow tract conduit early results from the U.S. Clinical trial, J. Am. Coll. Cardiol. 54 (2009) 1722-1729.

[6] K. Asoh, M. Walsh, E. Hickey, M. Nagiub, R. Chaturvedi, K.J. Lee, et al., Percutaneous pulmonary valve implantation within bioprosthetic valves, Eur. Heart J. 31 (2010) 1404-1409.

[7] D.B. McElhinney, W.E. Hellenbrand, E.M. Zahn, T.K. Jones, J.P. Cheatham, J.E. Lock, et al., Short- and medium-term outcomes after transcatheter pulmonary valve placement in the expanded multicenter US melody valve trial, Circulation 122 (2010) 507-516.

[8] M. Vezmar, R. Chaturvedi, K.J. Lee, C. Almeida, C. Manlhiot, B.W. McCrindle, et al. Percutaneous pulmonary valve implantation in the young 2-year follow-up, JACC Cardiovasc. Interv. 3 (2010) 439-448.

[9] A. Eicken, P. Ewert, A. Hager, B. Peters, S. Fratz, T. Kuehne, et al., Percutaneous pulmonary valve implantation: two-centre experience with more than 100 patients, Eur. Heart J. 32 (2011) 1260-1265.

[10] P. Lurz, A. Giardini, A.M. Taylor, J. Nordmeyer, V. Muthurangu, D. Odendaal, et al., Effect of altering pathologic right ventricular loading conditions by percutaneous pulmonary valve implantation on exercise capacity, Am. J. Cardiol. 105 (2010) 721-726.

[11] S. Hascoet, P. Acar, Y. Boudjemline, Transcatheter pulmonary valvulation: current indications and available devices, Arch. Cardiovasc. Dis. 107 (2014) 625-634.
[12] K.K. Mallula, D. Kenny, Z.M. Hijazi, Transjugular melody valve placement in a small child with protein losing enteropathy, Catheter. Cardiovasc. Interv. 85 (2015) 267-270.

[13] D.P. Berman, D.B. McElhinney, J.A. Vincent, W.E. Hellenbrand, E.M. Zahn, Feasibility and short-term outcomes of percutaneous transcatheter pulmonary valve replacement in small $(<30 \mathrm{~kg})$ children with dysfunctional right ventricular outflow tract conduits, Circ. Cardiovasc. Interv. 7 (2014) 142-148.

[14] Y. Boudjemline, S. Malekzadeh-Milani, M. Patel, J.B. Thambo, D. Bonnet, L. Iserin, et al., Predictors and outcomes of right ventricular outflow tract conduit rupture during percutaneous pulmonary valve implantation: a multicentre study, EuroIntervention 11 (2016) 1053-1062.

[15] R.J. Holzer, Z.M. Hijazi, Transcatheter pulmonary valve replacement: state of the art, Catheter. Cardiovasc. Interv. 87 (2016) 117-128.

[16] G. Habib, P. Lancellotti, M.J. Antunes, M.G. Bongiorni, J.P. Casalta, F. Del Zotti, et al., 2015 ESC Guidelines for the management of infective endocarditis: the Task Force for the Management of Infective Endocarditis of the European Society of Cardiology (ESC). Endorsed by: European Association for Cardio-Thoracic Surgery (EACTS), the European Association of Nuclear Medicine (EANM), Eur. Heart J. 36 (2015) 3075-3128.

[17] W. Finch, D.S. Levi, M. Salem, A. Hageman, J. Aboulhosn, Transcatheter melody valve placement in large diameter bioprostheses and conduits: what is the optimal "Landing zone"? Catheter. Cardiovasc. Interv. 86 (2015) E217-23.

[18] A. Fraisse, A. Assaidi, L. Mauri, S. Malekzadeh-Milani, J.B. Thambo, D. Bonnet, et al. Coronary artery compression during intention to treat right ventricle outflow with percutaneous pulmonary valve implantation: incidence, diagnosis, and outcome, Catheter. Cardiovasc. Interv. 83 (2014) E260-8.

[19] B.H. Morray, D.B. McElhinney, J.P. Cheatham, E.M. Zahn, D.P. Berman, P.M. Sullivan, et al., Risk of coronary artery compression among patients referred for transcatheter pulmonary valve implantation: a multicenter experience, Catheter. Cardiovasc. Interv. 6 (2013) 535-542.

[20] R.N. Bishnoi, T.K. Jones, J. Kreutzer, R.E. Ringel, NuMED Covered Cheatham-Platinum Stent for the treatment or prevention of right ventricular outflow tract conduit disruption during transcatheter pulmonary valve replacement, Catheter. Cardiovasc. Interv. 85 (2015) 421-427.

[21] A. Chatterjee, N.S. Bajaj, W.S. McMahon, M.G. Cribbs, J.S. White, A. Mukherjee, et al. Transcatheter pulmonary valve implantation: a comprehensive systematic review and meta-analyses of observational studies, J. Am. Heart Assoc. 6 (2017) https:// doi.org/10.1161/JAHA.117.006432. 